\title{
Psychische Auffälligkeiten von Jugendlichen mit Adipositas in der stationären Rehabilitation
}

\author{
Petra Hampel ${ }^{1}$, Franz Petermann $\dagger^{2}$ und Rainer Stachow ${ }^{3}$ \\ ${ }^{1}$ Europa-Universität Flensburg, Abteilung Gesundheitspsychologie und -bildung \\ ${ }^{2} Z$ entrum für Klinische Psychologie und Rehabilitation, Universität Bremen \\ ${ }^{3}$ Ostseeklinik Grömitz
}

\begin{abstract}
Zusammenfassung: Es wurden die Geschlechts- und Altersunterschiede in den selbst- und fremdbeurteilten psychischen Auffälligkeiten bei $N=142$ Jugendlichen mit Adipositas in der stationären Rehabilitation untersucht. Mädchen wiesen im Selbsturteil höhere Ausprägungen in der Ängstlichkeit/Depressivität sowie den emotionalen und Verhaltensproblemen auf. Die Eltern gaben eine höhere Hyperaktivität ihrer Söhne an. Jüngere schätzten ihre Verhaltensprobleme höher ein als Ältere. Zudem wurde die klinische Stichprobe mit geschlechts- und altersgematchten Kontrollen aus der Normierungsstudie des Screenings psychischer Störungen im Jugendalter (SPS-J-II; Hampel \& Petermann, 2012a) verglichen $(N=254)$. Es ergab sich ein 2.43-fach erhöhtes Risiko für auffällige Werte in der Ängstlichkeit/Depressivität bei den Jugendlichen mit Adipositas und ein signifikanter Mittelwertunterschied. Die Befunde sprechen für eine frühzeitige Diagnostik psychischer Auffälligkeiten und bedarfsgerechte Interventionen, um der Entwicklung komorbider psychischer Auffälligkeiten vorzubeugen.
\end{abstract}

Schlüsselwörter: Psychische Auffälligkeiten, Adipositas, Jugendliche, Geschlechts- und Altersunterschiede

\section{Psychological Problems Among Adolescents With Obesity in Inpatient Rehabilitation}

Abstract: Prior meta-analytic studies have shown that mental health is impaired among children and adolescents with obesity compared with normative data or healthy controls. Especially associations between obesity and internalizing problems have been found, which were smaller in self-reports than proxy reports. Moreover, some evidence has been provided in those studies that the mental problems are linked with adverse compliant behavior, particularly in adolescents with obesity. However, assessment of mental problems among adolescents with obesity in German inpatient rehabilitation has been widely neglected. Furthermore, comparisons of prevalence rates for mental problems with current German normative data are still pending. The present study investigated sex and developmental differences in mental problems among adolescents with obesity (body mass index > 97th percentile). Adolescents $(N=142)$ aged from 12 to 17 years in inpatient rehabilitation filled in the subscale emotional distress of the German version of the Reynolds Adolescent Adjustment Screening Inventory (RAASI; Hampel \& Petermann, 2012a). Moreover, adolescents and their parents were asked to complete the Strengths and Difficulties Questionnaire (SDQ; Woerner et al., 2002). Female adolescents reported higher levels of emotional distress on the RAASI as well as emotional and behavioral problems on the SDQ. Parents evaluated the hyperactivity of their sons as higher. Younger adolescents aged from 12 to 14 years scored higher on behavioral problems than the older group aged from 15 to 17 years. Furthermore, a clinical sample $(n=127)$ aged from 12 to 16 years was compared with sex- and age-matched controls in a normative study with the RAASI. Adolescents with obesity had a 2.43 -fold increased risk for clinical scores of emotional distress ( $95 \%$ confidence interval, $1.31-4.5)$ and scored on average higher on emotional distress than the normative sample. The results supported the early identification of mental problems among adolescents with obesity in inpatient rehabilitation and the application of needs-adjusted interventions to prevent the development or persistence of mental health problems.

Keywords: psychological problems, obesity, adolescents, effects of sex and age

Aktuelle epidemiologische Studien stellten eine Prävalenz für psychische Auffälligkeiten im Kindes- und Jugendalter von um die $20 \%$ fest (Barkmann \& Schulte-Markwort, 2012; Hölling, Schlack, Petermann, Ravens-Sieberer \& Mauz, 2014), die sich über die letzten 50 Jahre nicht signifikant verändert hat (Barkmann \& Schulte-Markwort, 2004). Gleichfalls wurde untermauert, dass die psychischen Auffälligkeiten zu 66 bis $78 \%$ bis ins Erwachse- nenalter persistieren (Lambert et al., 2013) sowie eine hohe globale Krankheitslast und Kosten verursachen (Karow et al., 2013). Ferner wurden ungünstige Auswirkungen auf verschiedenste Lebensbereiche (Klasen, Meyrose, Otto, Reiss \& Ravens-Sieberer, 2017) und die gesundheitsbezogene Lebensqualität belegt (Baumgarten et al., 2019). Chronisch körperliche Erkrankungen wie Adipositas hingen in Meta-Analysen mit psychischen 
Auffälligkeiten bei Kindern und Jugendlichen zusammen, insbesondere mit internalisierenden Auffälligkeiten (Pinquart, 2017), die wiederum in einer klinischen Studie ein Risikofaktor für eine geringere Compliance waren (Stachow, Kiera, Tiedjen \& Petermann, 2009). Bislang liegen noch wenige Studien zur Häufigkeit von psychischen Auffälligkeiten bei Jugendlichen mit Adipositas vor, die mit aktuellen deutschen Normen verglichen wurden. Dementsprechend sollten in der vorliegenden Studie deskriptive Befunde zu den Prävalenzen ermittelt, Geschlechts- und Altersunterschiede in den psychischen Auffälligkeiten bestimmt und ein Vergleich mit einer Normierungsstudie für die Ängstlichkeit/Depressivität angestellt werden.

\section{Psychische Auffälligkeiten im Jugendalter}

Neuere epidemiologische Studien, vor allem im Rahmen des Kinder- und Jugendgesundheitssurveys (KiGGS; Ravens-Sieberer, Klasen \& Petermann, 2016), verwenden für das Screening psychischer Auffälligkeiten häufig den Strengths and Difficulties Questionnaire (SDQ-Deu; Woerner et al., 2002), der über 25 Items folgende fünf Subtests erfasst: emotionale Probleme, Verhaltensprobleme, Hyperaktivität, Verhaltensprobleme mit Gleichaltrigen und prosoziales Verhalten. Insgesamt ergaben sich in der Basiserhebung des KiGGS auffällige Werte im Elternurteil für $9.1 \%$ in den emotionalen Problemen, $14.8 \%$ in den Verhaltensproblemen, $7.9 \%$ in der Hyperaktivität, $11.5 \%$ in den Verhaltensproblemen mit Gleichaltrigen und 3.6\% im prosozialen Verhalten (Hölling, Erhart, Ravens-Sieberer \& Schlack, 2007). Auch störungsspezifische PunktPrävalenzen der BELLA-Studie, dem Modul „Psychische Gesundheit" des KiGGS, lagen im Elternurteil in der Erhebungswelle 1 um die 10\% für Depression bzw. Angst, $5.7 \%$ bei Aufmerksamkeitsdefizit-Hyperaktivitätsstörungen (ADHS) sowie 12.2\% für Störungen des Sozialverhaltens (Klasen et al., 2016). Dagegen deuten einige Studien an, dass Abweichungen im Selbsturteil vom Elternurteil bestehen; mit eher höheren Angaben bei den internalisierenden Auffälligkeiten und geringeren Ausprägungen bei der ADHS (Klasen et al., 2016).

Diese Prävalenzen unterschieden sich jedoch insbesondere in Abhängigkeit von den Risikofaktoren Geschlecht und Alter: Hinsichtlich des Geschlechts konnte die Evidenz erbracht werden, dass Mädchen höhere Ausprägungen in den emotionalen Problemen bzw. der Depression und Angst aufweisen (Becker et al., 2018; Hölling et al., 2014; Klasen et al., 2016; Lohbeck, Schultheiß, Peter- mann \& Petermann, 2015). Demgegenüber zeigten sich bei Jungen konsistent höhere Ausprägungen in den Verhaltensproblemen bzw. Störungen des Sozialverhaltens (Hölling et al., 2014; Klasen et al., 2016; Lohbeck et al., 2015), aber auch in der Hyperaktivität bzw. ADHS (Hölling et al., 2014; Klasen et al., 2016). Diese Geschlechtsunterschiede wurden für das Selbsturteil in einer großangelegten britischen Studie mit Jugendlichen zwischen 11 und 14 Jahren bestätigt (Deighton et al., 2019). Befunde zu den Altersunterschieden sind eher heterogen. Emotionale und Verhaltensprobleme waren sowohl bei Älteren erhöht (Becker et al., 2018; Deighton et al., 2019), als auch bei 7- bis 13-Jährigen bzw. 3- bis 13-Jährigen (Hölling et al., 2007).

In der vorliegenden Studie wurde zusätzlich zum SDQDeu im Selbst- und Elternurteil noch die selbstberichtete Ängstlichkeit/Depressivität anhand des Screenings psychischer Störungen im Jugendalter (SPS-J-II; Hampel \& Petermann, 2012a) erfasst, für die in einer psychiatrischen Inanspruchnahmepopulation eine sehr gute Sensitivität nachgewiesen wurde (Goldbeck, Besier, Petermann, Karpinski \& Hampel, 2007) und sich erhöhte Ausprägungen bei Mädchen und bei der älteren Gruppe der 14- bis 16Jährigen ergaben (Hampel \& Petermann, 2012b).

\section{Psychische Auffälligkeiten bei chronisch körperlichen Erkrankungen}

Chronisch körperliche Erkrankungen im Kindes- und Jugendalter gingen allgemein in Studien mit höheren psychischen Auffälligkeiten einher. So ergaben sich insbesondere erhöhte Ausprägungen in den internalisierenden Auffälligkeiten mit kleinen Effektstärken beim Selbsturteil und mäßigen Effektstärken für das Elternurteil (Pinquart, 2017). In einer gesonderten Meta-Analyse zum $\mathrm{Zu}$ sammenhang mit depressiven Symptomen wiesen die klinischen Stichproben höhere Werte als die gesunden Kontrollen auf, wobei insbesondere Mädchen betroffen waren und sich höhere Effekte in den Elternurteilen als im Selbsturteil ergaben (Pinquart \& Shen, 2011b). Höhere Angstsymptome wurden in einer weiteren Meta-Analyse nachgewiesen (Pinquart \& Shen, 2011a).

In der klinischen Studie von Stachow et al. (2009) bei 179 Jugendlichen mit chronisch körperlichen Erkrankungen im Alter zwischen 14 und 17 Jahren wurden alle vier Subtests des SPS-J eingesetzt: aggressiv-dissoziales Verhalten, Ärgerkontrollprobleme, Ängstlichkeit/Depressivität und Selbstwertprobleme. Es konnte anhand der Normen der ersten Auflage des SPS-J festgestellt werden, 
dass $33.5 \%$ der Jugendlichen klinisch auffällige Werte in mindestens einem Subtest aufwiesen. Hierbei gaben Mädchen außer in den Ärgerkontrollproblemen in den anderen drei Subtests höhere Ausprägungen als die Jungen an. Alterseffekte konnten jedoch nicht abgesichert werden.

\section{Psychische Auffälligkeiten bei Adipositas}

Insgesamt wurden Kinder und Jugendliche mit Adipositas wenig untersucht. Pinquart (2017, S. 663) zieht folgendes Fazit aus meta-analytischen Befunden: „Kinder und Jugendliche mit Adipositas wiesen kleine signifikante Erhöhungen von Depressivität, Angst und externalisierenden Problemen sowie mäßig erhöhte internalisierende Probleme aus Elternsicht auf". In einer früheren Studie mit einem Datensatz der Gmündner ErsatzKasse über $N=156.948$ 6- bis 14-Jährige mit ärztlich diagnostizierter Adipositas war das Risiko zweifach erhöht, komorbid internalisierende Störungen aufzuweisen (Eschenbeck, Kohlmann, Dudey \& Schurholz, 2009). Für die einzelnen Störungsbereiche betrug die odds ratio $(O R)$ für Angststörungen 1.74 und für Depression 3.21. Ein zweifach erhöhtes Risiko war für die externalisierenden Störungen nachweisbar $(O R=1.64$; ADHS: 1.43 , Störungen des Sozialverhaltens: 2.17). Hierbei zeigten sich besonders für die internalisierenden Störungen, und hier für die Angststörungen, erhöhte Risiken bei den Mädchen und bei der älteren Gruppe der 12- bis 14-Jährigen. Auch in der KiGGS Welle 1 konnten anhand des SDQ erhöhte selbstberichtete psychische Auffälligkeiten bei 6.813 Jugendlichen im Alter zwischen 11 und 17 Jahren festgestellt werden (Krause, Kleiber \& Lampert, 2014): Übergewicht war mit einem 1.4-fach erhöhten Risiko und Adipositas mit einem 2.5-fach erhöhten Risiko für psychische Auffälligkeiten verbunden.

Hinsichtlich der Wirkungsrichtung liegen noch widersprüchliche Befunde vor, allerdings sprechen aktuelle Befunde einer Längsschnittstudie für eine bidirektionale Beziehung zwischen Adipositas und Depression (Mannan, Mamun, Doi \& Clavarino, 2016). Insgesamt belegten aktuelle internationale Meta-Analysen den Zusammenhang zwischen Adipositas und insbesondere der Depression oder den depressiven Symptomen (Quek, Tam, Zhang \& Ho, 2017; Wang et al., 2019). Aber Depression hat sich auch als Risikofaktor für die Entstehung (Chao, Wadden \& Berkowitz, 2019) und Aufrechterhaltung einer Adipositas (vgl. Sagar \& Gupta, 2018) erwiesen.
In der vorliegenden Arbeit wurden zwei Fragestellungen verfolgt: Zunächst wurden die psychischen Auffälligkeiten der Jugendlichen mit Adipositas in Abhängigkeit des Geschlechts und Alters untersucht. Des Weiteren wurde die Ängstlichkeit/Depressivität dieser klinischen Gruppe von Jugendlichen mit Adipositas mit geschlechtsund altersgematchten Jugendlichen der Normstichprobe des SPS-J-II verglichen.

Die Fragestellungen wurden anhand von Querschnittdaten einer klinischen Stichprobe von Jugendlichen überprüft, die an einer stationären Rehabilitation in der Fachklinik Sylt für Kinder und Jugendliche der Deutschen Rentenversicherung (DRV) Nord im Zeitraum von Juni 2016 bis Mai 2017 teilnahmen. Die in der zweiten Auflage aktualisierten Normen des SPS-J-II (Hampel \& Petermann, 2012a) wurden an einer Stichprobe im Herbst 2011 gewonnen.

\section{Fragestellung 1: Geschlechts- und Altersunterschiede in der klinischen Stichprobe}

\section{Methode}

\section{Ein- und Ausschlusskriterien}

Es wurden Jugendliche ohne Begleitpersonen in die Stichprobe eingeschlossen, die zwischen 12 und 17 Jahre alt waren. Hauptdiagnose musste dabei eine Adipositas sein (Body-Mass-Index, BMI > 97. Perzentile). Ferner waren ausreichende Deutschkenntnisse erforderlich, die sich auf die Lese- und Schreibfähigkeiten bezogen. Ausgeschlossen wurden Jugendliche, die weitere psychiatrische Begleiterkrankungen aufwiesen oder keine Bereitschaft zur Teilnahme zeigten.

Für die Studie lag ein positives Ethikvotum der Deutschen Gesellschaft für Psychologie vor (PH_112015). Die Deklaration von Helsinki wurde eingehalten. Die Eltern und Jugendlichen gaben eine schriftliche Einverständniserklärung zu Beginn der Studie.

\section{Design und Stichprobe}

Die Fragestellung wurde anhand eines zweifaktoriellen Designs mit einer konsekutiven Stichprobe von $N=142$ Jugendlichen untersucht: Die unabhängigen Variablen wurden durch das Geschlecht (weiblich vs. männlich) und die Altersgruppe (12-14 vs. 15-17 Jahre) gebildet (weiblich, 12-14 J.: $n=48$; weiblich, 15-17 J.: $n=39$; männlich, 12-14 J.: $n=36$; männlich, 15-17 J.: $n=19$; Geschlecht ${ }^{\star}$ Altersgruppe: $\left.\chi^{2}(1)=1.47, \mathrm{~ns}\right)$. 
$\mathrm{Zu}$ Beginn der Rehabilitation wiesen die Jugendlichen ein mittleres Alter von 14.18 Jahren $(S D=1.46)$ und einen mittleren BMI-standard deviation score (BMI-SDS) von $2.67(S D=0.58)$ auf. Zudem besuchten sie mehrheitlich die Gesamtschule $(n=47,33.8 \%)$ oder Realschule $(n=40,28.8 \%)$. Ihre Eltern waren mehrheitlich verheiratet $(n=70,56.5 \%)$.

\section{Messinstrumente}

Emotionale Auffälligkeiten. Die emotionalen Auffälligkeiten wurden mit den zehn Items des Subtests „Ängstlichkeit/Depressivität" des SPS-J-II (Hampel \& Petermann, 2012a) erfasst. Die Items wurden auf einer dreistufigen Häufigkeitsskalierung von ,nie oder fast nie“ (0) bis "fast immer" (2) in Bezug auf die letzten sechs Monate eingeschätzt. Die Ängstlichkeit/Depressivität variierte von 0 bis 20. Die interne Konsistenz betrug .87 .

Psychische Auffälligkeiten. Um psychische Auffälligkeiten im Selbsturteil zu erfassen, wurde die deutsche Fassung des SDQ-Deu (SDQ-S; Woerner et al., 2002) für Kinder und Jugendliche zwischen 11 und 18 Jahre verwendet. Die Eltern bearbeiteten den Fragebogen in der Version SDQ-Deu Fremd 4-17. Die Aussagen der 25 Items wurden von "nicht zutreffend“ (0) bis "eindeutig zutreffend" (2) eingeschätzt. Die fünf positiv formulierten Items der Problemskalen wurden entsprechend umgepolt. In der vorliegenden Arbeit wurden die fünf Subtests herangezogen. Die Subtestwerte können zwischen 0 und 10 variieren. Bei den Eltern fehlten für die emotionalen Probleme vier Angaben $(N=138)$ und in den anderen Subtests jeweils fünf $(N=137)$. Während die internen Konsistenzen der Elternangaben befriedigend waren, lagen die Cronbach Alphas des Selbsturteils im unbefriedigenden Bereich, insbesondere in den drei Subtests mit umzupolenden Items. Die kritischen Werte für auffällige Ausprägungen (Cut-off-Werte) für die Selbsteinschätzung wurden nach Becker et al. (2018) bestimmt und für das Elternurteil nach Woerner et al. (2002).

\section{Statistische Auswertung}

Die Unterschiede in Abhängigkeit vom Geschlecht und Alter in den emotionalen Auffälligkeiten wurden anhand einer univariaten zweifaktoriellen Varianzanalyse mit den beiden Zwischen-Subjekt-Faktoren Geschlecht (weiblich vs. männlich) und Altersgruppe (12-14 vs. 15-17 Jahre) überprüft. Zudem wurden für die psychischen Auffälligkeiten gemessen über den SDQ-Selbst und SDQ-Eltern zwei multivariate zweifaktorielle Varianzanalysen jeweils über die fünf Subtests berechnet und univariate zweifaktorielle Varianzanalysen angeschlossen. Insgesamt wurden für alle Subtests Bonferroni-adjustierte $t$-tests gerechnet. Das Signifikanzniveau für die uni- und multivariaten Testungen im Rahmen der Varianzanalyse wurde aufgrund des explorativen Charakters der Studie auf $p<.05$ festgesetzt. Klinische Effektstärken wurden anhand von Cohens $d$ berechnet mit $d=.20$ als kleine, $d=$ .50 als mittlere und $d=.80$ als große Effektstärke (Cohen, 1988). Eine Varianzaufklärung von über $\eta^{2}=.01$ wurde als kleine, über $\eta^{2}=.06$ als mittlere und über $\eta^{2}=.14$ als große Effektstärke bewertet (Bühner \& Ziegler, 2012).

\section{Ergebnisse}

\section{Deskriptive Ergebnisse}

Zunächst sollen die Häufigkeitsverteilungen der psychischen Auffälligkeiten beschrieben werden (Tab. 1). Knapp ein Drittel der Jugendlichen zeigte auffällige Werte in der Ängstlichkeit/Depressivität. Die psychischen Auffälligkeiten im Selbsturteil verteilten sich wie folgt: Auffällige Werte wurden in den emotionalen Problemen bei $29.6 \%$, in den Verhaltensproblemen bei $74.6 \%$, in den Problemen mit Gleichaltrigen bei $75.4 \%$ und im prosozialen Verhalten bei $56.3 \%$ festgestellt. In der Hyperaktivität lag lediglich ein Fall über dem Cut-off-Wert. Die Eltern beurteilten die emotionalen Probleme ihrer Kinder mit $19.0 \%$ als auffällig, die Verhaltensprobleme mit $17.6 \%$, die Hyperaktivität mit 7.0\%, die Probleme mit Gleichaltrigen mit $15.5 \%$ und das prosoziale Verhalten bei $4.2 \%$.

Tabelle 1. Verteilung der psychischen Auffälligkeiten

\begin{tabular}{lccc}
\hline Kennwert & $\begin{array}{c}\text { unauffällig } \\
\text { Anzahl (\%) }\end{array}$ & $\begin{array}{c}\text { grenzwertig } \\
\text { Anzahl (\%) }\end{array}$ & $\begin{array}{c}\text { auffällig } \\
\text { Anzahl (\%) }\end{array}$ \\
\hline SPS-J-II & & & \\
AD & $100(70.4)$ & $/$ & $42(29.6)$ \\
\hline SDQ-Selbsturteil & & & \\
EMO-S & $71(50.0)$ & $29(20.4)$ & $106(74.6)$ \\
VER-S & $13(09.2)$ & $23(16.2)$ & $1(00.7)$ \\
HYP-S & $138(97.2)$ & $3(02.1)$ & $107(75.4)$ \\
GL-S & $13(09.2)$ & $22(15.5)$ & $80(56.3)$ \\
SOZ-S & $24(16.9)$ & $38(26.8)$ & $27(19.0)$ \\
SDQ-Elternurteil & & & $25(17.6)$ \\
EMO-E & $99(69.7)$ & $12(08.5)$ & $10(07.0)$ \\
VER-E & $97(68.3)$ & $17(12.0)$ & $22(15.5)$ \\
HYP-E & $113(79.6)$ & $16(11.3)$ & $6(04.2)$ \\
GL-E & $88(62.0)$ & $29(20.4)$ & $6(04.2)$ \\
SOZ-E & $127(89.4)$ & & \\
\hline
\end{tabular}

Anmerkungen: SPS-J-II = Screening psychischer Störungen im Jugendalter, $\mathrm{SDQ}=$ Strengths and Difficulties Questionnaire, $\mathrm{AD}=$ Ängstlichkeit/Depressivität; $\mathrm{S}=$ Selbsturteil, $\mathrm{E}=$ Elternurteil; $\mathrm{EMO}=$ Emotionale Probleme, VER $=$ Verhaltensprobleme, $\mathrm{HYP}=$ Hyperaktivität, $\mathrm{GL}=$ Verhaltensprobleme mit Gleichaltrigen, $\mathrm{SOZ}=$ Prosoziales Verhalten 
Tabelle 2. Mittelwerte (M), Standardfehler (SE) und Ergebnisse der univariaten Varianzanalysen mit der F-Statistik für die Haupteffekte „Geschlecht" und „Altersgruppe" und deren Wechselwirkung

\begin{tabular}{|c|c|c|c|c|c|c|c|c|c|}
\hline \multirow[b]{3}{*}{ Kennwert } & & & & & & & \multicolumn{3}{|c|}{ Faktor } \\
\hline & & \multicolumn{2}{|c|}{ Weiblich } & \multicolumn{2}{|c|}{ Männlich } & & \multirow{2}{*}{$\begin{array}{c}\text { Geschlecht (A) } \\
F(1,138)\end{array}$} & \multirow{2}{*}{$\begin{array}{l}\text { Alter (B) } \\
F(1,138)\end{array}$} & \multirow{2}{*}{$\begin{array}{c}A \times B \\
F(1,138)\end{array}$} \\
\hline & & $12-14$ & $15-17$ & $12-14$ & $15-17$ & & & & \\
\hline \multicolumn{10}{|l|}{ SPS-J-II } \\
\hline$A D$ & $\begin{array}{l}M \\
S E\end{array}$ & $\begin{array}{l}7.81 \\
0.66\end{array}$ & $\begin{array}{l}8.26 \\
0.74\end{array}$ & $\begin{array}{l}6.36 \\
0.76\end{array}$ & $\begin{array}{l}4.74 \\
1.05\end{array}$ & $\begin{array}{c}F \\
\eta 2\end{array}$ & $\begin{array}{c}9.23 * \star \\
.063\end{array}$ & $\begin{array}{l}0.52 \\
.004\end{array}$ & $\begin{array}{l}1.60 \\
.011\end{array}$ \\
\hline \multicolumn{10}{|c|}{ SDQ-Selbsturteil } \\
\hline EMO-S & $\begin{array}{l}M \\
S E\end{array}$ & $\begin{array}{l}5.06 \\
0.23\end{array}$ & $\begin{array}{l}4.82 \\
0.26\end{array}$ & $\begin{array}{l}4.53 \\
0.27\end{array}$ & $\begin{array}{l}4.16 \\
0.37\end{array}$ & $\begin{array}{c}F \\
\eta 2\end{array}$ & $\begin{array}{c}4.28 * \\
.030\end{array}$ & $\begin{array}{l}1.12 \\
.008\end{array}$ & $\begin{array}{l}0.05 \\
.000\end{array}$ \\
\hline VER-S & $\begin{array}{l}M \\
S E\end{array}$ & $\begin{array}{l}5.58 \\
0.19\end{array}$ & $\begin{array}{l}5.21 \\
0.21\end{array}$ & $\begin{array}{l}5.17 \\
0.22\end{array}$ & $\begin{array}{l}4.05 \\
0.30\end{array}$ & $\begin{array}{c}F \\
\eta 2\end{array}$ & $\begin{array}{c}11.45^{\star \star} \\
.077\end{array}$ & $\begin{array}{c}10.35^{\star \star} \\
.070\end{array}$ & $\begin{array}{l}2.52 \\
.018\end{array}$ \\
\hline HYP-S & $\begin{array}{l}M \\
S E\end{array}$ & $\begin{array}{l}3.15 \\
0.17\end{array}$ & $\begin{array}{l}3.21 \\
0.19\end{array}$ & $\begin{array}{l}3.42 \\
0.19\end{array}$ & $\begin{array}{l}3.05 \\
0.27\end{array}$ & $\begin{array}{c}F \\
\eta^{2}\end{array}$ & $\begin{array}{l}0.08 \\
.001\end{array}$ & $\begin{array}{l}0.55 \\
.004\end{array}$ & $\begin{array}{l}1.06 \\
.008\end{array}$ \\
\hline GL-S & $\begin{array}{l}M \\
S E\end{array}$ & $\begin{array}{l}5.35 \\
0.18\end{array}$ & $\begin{array}{l}5.18 \\
0.20\end{array}$ & $\begin{array}{l}5.11 \\
0.21\end{array}$ & $\begin{array}{l}5.05 \\
0.29\end{array}$ & $\begin{array}{c}F \\
\eta 2\end{array}$ & $\begin{array}{l}0.67 \\
.005\end{array}$ & $\begin{array}{l}0.27 \\
.002\end{array}$ & $\begin{array}{l}0.07 \\
.000\end{array}$ \\
\hline SOZ-S & $\begin{array}{l}M \\
S E\end{array}$ & $\begin{array}{l}4.94 \\
0.23 \\
\end{array}$ & $\begin{array}{l}5.23 \\
0.25 \\
\end{array}$ & $\begin{array}{l}4.36 \\
0.26 \\
\end{array}$ & $\begin{array}{l}4.74 \\
0.36 \\
\end{array}$ & $\begin{array}{c}F \\
\eta 2\end{array}$ & $\begin{array}{l}3.63 \\
.026 \\
\end{array}$ & $\begin{array}{l}1.42 \\
.010 \\
\end{array}$ & $\begin{array}{l}0.02 \\
.000 \\
\end{array}$ \\
\hline \multicolumn{10}{|c|}{ SDQ-Elternurteil } \\
\hline EMO-Ea & $\begin{array}{l}M \\
S E\end{array}$ & $\begin{array}{l}3.60 \\
0.36\end{array}$ & $\begin{array}{l}4.41 \\
0.39\end{array}$ & $\begin{array}{l}4.35 \\
0.42\end{array}$ & $\begin{array}{l}4.39 \\
0.58\end{array}$ & $\begin{array}{c}F \\
\eta 2\end{array}$ & $\begin{array}{l}0.68 \\
.005\end{array}$ & $\begin{array}{l}0.91 \\
.007\end{array}$ & $\begin{array}{l}0.76 \\
.006\end{array}$ \\
\hline VER-E ${ }^{a}$ & $\begin{array}{l}M \\
S E\end{array}$ & $\begin{array}{l}2.79 \\
0.28\end{array}$ & $\begin{array}{l}2.59 \\
0.31\end{array}$ & $\begin{array}{l}2.53 \\
0.33\end{array}$ & $\begin{array}{l}2.67 \\
0.46\end{array}$ & $\begin{array}{c}F \\
\eta 2\end{array}$ & $\begin{array}{l}0.07 \\
.000\end{array}$ & $\begin{array}{l}0.01 \\
.000\end{array}$ & $\begin{array}{l}0.23 \\
.002\end{array}$ \\
\hline HYP-E & $\begin{array}{l}M \\
S E\end{array}$ & $\begin{array}{l}3.28 \\
0.32\end{array}$ & $\begin{array}{l}2.90 \\
0.36\end{array}$ & $\begin{array}{l}4.50 \\
0.38\end{array}$ & $\begin{array}{l}4.00 \\
0.52\end{array}$ & $\begin{array}{c}F \\
\eta^{2}\end{array}$ & $\begin{array}{c}8.36^{\star *} \\
.059\end{array}$ & $\begin{array}{l}1.19 \\
.009\end{array}$ & $\begin{array}{l}0.02 \\
.000\end{array}$ \\
\hline$G L-E^{a}$ & $\begin{array}{l}M \\
S E\end{array}$ & $\begin{array}{l}2.79 \\
0.34\end{array}$ & $\begin{array}{l}3.31 \\
0.37\end{array}$ & $\begin{array}{l}3.29 \\
0.40\end{array}$ & $\begin{array}{l}3.50 \\
0.55\end{array}$ & $\begin{array}{c}F \\
\eta^{2}\end{array}$ & $\begin{array}{l}0.68 \\
.005\end{array}$ & $\begin{array}{l}0.74 \\
.005\end{array}$ & $\begin{array}{l}0.14 \\
.001\end{array}$ \\
\hline$S O Z-E^{a}$ & $\begin{array}{l}M \\
S E\end{array}$ & $\begin{array}{l}8.36 \\
0.26\end{array}$ & $\begin{array}{l}8.10 \\
0.28\end{array}$ & $\begin{array}{l}7.74 \\
0.30\end{array}$ & $\begin{array}{l}7.89 \\
0.42\end{array}$ & $\begin{array}{c}F \\
\eta 2\end{array}$ & $\begin{array}{l}1.73 \\
.013\end{array}$ & $\begin{array}{l}0.03 \\
.000\end{array}$ & $\begin{array}{l}0.42 \\
.003\end{array}$ \\
\hline
\end{tabular}

Anmerkungen: SPS-J-II = Screening psychischer Störungen im Jugendalter, SDQ = Strengths and Difficulties Questionnaire, AD = Ängstlichkeit/Depressivität; $S=$ Selbsturteil, E = Elternurteil; EMO = Emotionale Probleme, VER = Verhaltensprobleme, HYP = Hyperaktivität, GL = Verhaltensprobleme mit Gleichaltrigen, $\mathrm{SOZ}=$ Prosoziales Verhalten; $F=$ Prüfgröße, $\eta^{2}=$ Eta-Quadrat. ${ }^{*} p<.05 ; * \star p<.01 .{ }^{a}=$ df2 $=134$.

\section{Inferenzstatistische Ergebnisse}

Emotionale Auffälligkeiten. In der univariaten zweifaktoriellen Varianzanalyse ergab sich ein signifikanter Haupteffekt „Geschlecht" mit kleiner Effektstärke (Tab. 2). Mädchen zeigten eine höhere Ängstlichkeit/ Depressivität auf als Jungen.

Psychische Auffälligkeiten - Selbst. In der multivariaten zweifaktoriellen Varianzanalyse war sowohl ein signifikanter Haupteffekt "Geschlecht“ als auch ein signifikanter Haupteffekt „Alter“ mit mittleren Effektstärken nachweisbar $\left(F_{\text {Geschlecht }}(5,134)=3.07, p=.012\right.$, $\eta^{2}=.103 ; F_{\text {Alter }}(5,134)=2.76, p=.021, \eta^{2}=.093 ; F_{G e}$ schlecht $x$ Alter $\left.(5,134)=0.73, p=.605, n s, \eta^{2}=.026\right)$. Hinsichtlich des Geschlechtsunterschiedes zeigten sich signifikant höhere Ausprägungen in den emotionalen und Verhaltensproblemen bei den Mädchen mit einer kleinen bzw. mittleren Effektstärke (Tab. 2). Darüber hinaus wiesen Jüngere signifikant höhere Verhaltens- probleme auf als Ältere, wobei sich eine mittlere Effektstärke ergab.

Psychische Auffälligkeiten - Fremd. In der zweiten multivariaten zweifaktoriellen Varianzanalyse war ein signifikanter Haupteffekt "Geschlecht“ mit mittleren Effektstärken feststellbar $\left(F_{\text {Geschlecht }}(5,130)=3.09, p=\right.$ $.011, \eta^{2}=.106 ; F_{\text {Alter }}(5,130)=0.62, p=.685, n s, \eta^{2}$ $=.023 ; F_{\text {Geschlecht } x \text { Alter }}(5,130)=0.49, p=.787, n s, \eta^{2}$ $=.018$ ). Für die Jungen ergaben sich signifikant höhere Ausprägungen in der Hyperaktivität, was durch eine kleine Effektstärke gekennzeichnet war. 


\section{Fragestellung 2: Normvergleich in den emotionalen Auffälligkeiten}

\section{Methode}

\section{Ein- und Ausschlusskriterien}

Um die Stichproben vergleichbar zu gestalten, wurden aus der klinischen Stichprobe alle Jugendlichen im Alter von 17 Jahren ausgeschlossen $(n=14)$. Außerdem musste für die Angleichung der Gruppen ein 16-jähriges Mädchen per Zufall ausgeschlossen werden, sodass die Experimentalgruppe (EG) $n=127$ Jugendliche umfasste. In der Normierungsstichprobe des SPS-J-II über $N=1.079$ wurden zunächst alle Jugendlichen im Alter von 10 und 11 Jahren ausgeschlossen. $\mathrm{Zu}$ den Jugendlichen mit Adipositas wurden nach Geschlecht und Alter gematchte sowie nach Schulform parallelisierte Kontrollen (KG) aus der Normstichprobe gezogen. Die Eltern sollten in einem Fragebogen angeben, ob ihr Kind eine chronische Erkrankung (u.a. Adipositas) aufweist. Allerdings wurden die Elternfragebogen nicht von allen Eltern und nicht immer vollständig bearbeitet, sodass die Norm nicht als gesunde Kontrolle gelten kann.

\section{Design und Stichprobe}

Die Fragestellung zur Ängstlichkeit/Depressivität des SPS-JII wurde anhand eines einfaktoriellen Designs bei $N=254$ Jugendlichen untersucht: Die unabhängige Variable „Gruppe“ war zweifach abgestuft in "Jugendliche mit Adipositas“ (EG) und „Norm“ (KG).

In die jeweilige Teilstichprobe gingen 74 Mädchen (58.3\%) und 53 Jungen im Alter von 12 bis 16 Jahren ein. Beide Teilstichproben wiesen jeweils ein mittleres Alter von 13.85 Jahren $(S D=1.17)$ auf. Die Schulformen verteilten sich wie folgt: Hauptschule EG: $12.7 \%$, KG: $11.0 \%$, Realschule EG: $30.2 \%$, KG: $31.5 \%$, Gymnasium EG: 11.1\%, KG: 15\%, Gesamtschule EG: $35.7 \%$, KG: $37.0 \%$, sonstige EG: 10.3\%, KG: $5.5 \%$. Zu Beginn der Rehabilitation wiesen die Jugendlichen mit Adipositas einen mittleren BMI-SDS von 2.63 $(S D=0.54$; min: 1.17, max: 4.03) auf.

\section{Statistische Auswertung}

Die Unterschiede in der Ängstlichkeit/Depressivität der beiden Gruppen wurden mittels eines unabhängigen $t$-Tests überprüft. Die klinische Effektstärke wurde wiederum anhand von Cohens $d$ berechnet.

\section{Ergebnisse}

\section{Deskriptive Ergebnisse}

Die Jugendlichen mit Adipositas zeigten zu 29.9\% auffällige Werte in der Ängstlichkeit/Depressivität $(n=38)$. Dagegen lagen $15 \%$ der Jugendlichen der Normstichprobe über dem Cut-off ( $n=19,15.0 \%)$. Das Risikoverhältnis (Odds Ratio) lag bei 2.43 (95\%-Konfidenzintervall: 1.31-4.5).

\section{Inferenzstatistische Ergebnisse}

Der Levene-Test wies eine Varianzinhomogenität nach, sodass der Unterschied anhand des Welch-Tests überprüft wurde. Dieser stellte einen signifikanten Unterschied mit kleiner Effektstärke fest $(t(237.0)=2.35$, $p=.020, d=.29$ ). Die klinische Gruppe zeigte höhere Ausprägungen als die KG auf (EG: $M=7.09, S D=4.78$; KG: $M=5.83, S D=3.69$ ).

\section{Diskussion}

Psychische Auffälligkeiten im Kindes- und Jugendalter beeinträchtigen verschiedenste Lebensbereiche wie die schulische Leistungsfähigkeit oder soziale Beziehungen (Klasen et al., 2017). Eine aktuelle Auswertung des KiGGS Welle 2 erbrachte insbesondere eine geringere körperliche, psychische und elternbezogene Lebensqualität bei Jugendlichen mit einem auffälligen Gesamtproblemwert im SDQ-S (Baumgarten et al., 2019). Chronisch körperliche Erkrankungen gehen zudem auch mit psychischen Auffälligkeiten einher. So belegten Meta-Analysen ungünstige Werte chronisch körperlich kranker Kinder und Jugendlicher insbesondere in den internalisierenden Auffälligkeiten (Pinquart, 2017; Pinquart \& Shen, 2011a, 2011b). Aktuelle internationale Meta-Analysen konnten den Zusammenhang zwischen Adipositas und internalisierenden Auffälligkeiten bestätigen (Quek et al., 2017; Wang et al., 2019). Wenige deutsche Studien bei Kindern und Jugendlichen mit Adipositas der Allgemeinbevölkerung ergaben ähnliche Befunde (Eschenbeck et al., 2009; Krause et al., 2014). In der vorliegenden Studie sollten diese Befunde anhand neuerer Instrumente und Normen untersucht werden.

Die deskriptiven Statistiken unterstützen bisherige Befunde, dass die Häufigkeiten in der selbstberichteten Ängstlichkeit/Depressivität des SPS-J-II, aber auch in den emotionalen Problemen im SDQ-S mit jeweils 29.6\% auffälligen Werten erhöht waren. Die Angaben konnten sich somit über die beiden Selbsteinschätzungsskalen trotz sehr unterschiedlicher Reliabilitäten bestätigen. Da auch die anderen Subtests des SDQ-S unbefriedigende interne Konsistenzen aufwiesen, soll auf diese Befunde nicht eingegangen werden. Ein Vergleich der Elternangaben der Allgemeinbevölkerung von Hölling et al. (2007) zeigt, dass in der vorliegenden Stichprobe mit Adipositas die Häufigkeiten in den emotionalen Problemen deutlich erhöht sowie in den Verhaltensproblemen und den Pro- 
blemen im Umgang mit Gleichaltrigen erhöht sind. Vorbehaltlich der geringen Reliabilitäten im SDQ-S spricht der Vergleich der beiden Urteiler übereinstimmend mit Pinquart (2017) dafür, dass Kinder und Jugendliche mit chronisch körperlichen Erkrankungen ihre emotionalen Probleme höher einstufen als ihre Eltern. Diese geringere Wahrnehmung auf Seiten der Eltern konnte auch für die BELLA-Studie nachgewiesen werden (Klasen et al., 2016) und kann darauf zurückgeführt werden, dass Eltern die internalisierenden Prozesse eher übersehen (Rossmann \& Pichler-Janisch, 1998).

\section{Normvergleich in den emotionalen Auffälligkeiten}

In dem deskriptiven Vergleich mit der geschlechts- und altersgematchten KG, die aus der Normierungsstichprobe des SPS-J-II gezogen wurde, waren mit $30 \%$ der Jugendlichen mit Adipositas deutlich mehr auffällig als Jugendliche der KG, bei denen $15 \%$ auffällige Werte in der Ängstlichkeit/Depressivität zeigten. Dies entsprach einer $O R$ von 2.43 (95\%-Konfidenzintervall: 1.31-4.5). Dieser Befund überschreitet sogar Ergebnisse von Eschenbeck et al. (2009), die ein zweifach erhöhtes Risiko für internalisierende Störungen bei Kindern und Jugendlichen mit ärztlich diagnostizierter Adipositas der Allgemeinbevölkerung feststellten. Zudem gelangten Krause et al. (2014) zu ähnlichen Befunden in einer Stichprobe der Allgemeinbevölkerung, indem Jugendliche mit Adipositas ein 2.5-fach erhöhtes Risiko für auffällige Gesamtproblemwerte im SDQ-S aufwiesen. Demnach kann angenommen werden, dass trotz der Selektion in der vorliegenden klinischen Stichprobe im Vergleich zur Allgemeinbevölkerung die Prävalenz ähnlich ausfiel. Schließlich belegte der signifikante unabhängige $t$-Tests die erhöhten Ausprägungen in den emotionalen Auffälligkeiten der Jugendlichen mit Adipositas. Ähnlich zu Befunden von Meta-Analysen bei chronisch körperlichen Erkrankungen zeigte sich ein kleiner Effekt für das Selbsturteil (Pinquart, 2017).

\section{Geschlechts- und Altersunterschiede in der klinischen Stichprobe}

In Übereinstimmung mit früheren Befunden in der Allgemeinbevölkerung konnten die erhöhten Ausprägungen in der Ängstlichkeit/Depressivität und den emotionalen Problemen des SDQ-S bei den Mädchen bestätigt werden (Becker et al., 2018; Hampel \& Petermann, 2012a, 2012b; Hölling et al., 2007, 2014; Klasen et al., 2016; Lohbeck et al., 2015). Erklärungsansätze zum erhöhten Risiko von
Mädchen für eine Entwicklung von depressiven Symptomen betonen die multifaktorielle Entstehung (vertiefend s. Groen \& Petermann, 2011; zusammenfassend s. Thapar, Collishaw, Pine \& Thapar, 2012). So kommen genetische, hormonelle, neuronale und psychosoziale Risikofaktoren zum Tragen. Hierbei wird im Hinblick auf psychosoziale Faktoren insgesamt angenommen, dass Mädchen eine erhöhte Vulnerabilität aufweisen, weil sie eine emotionale und selbstkritischere Verarbeitung negativer Erfahrungen, eine höhere soziale Sensibilität und Verantwortungsübernahme bei erhöhten Belastungen durch zwischenmenschliche Probleme und Verluste besonders in der Pubertät aufweisen (Groen \& Petermann, 2011).

Die erhöhten Verhaltensprobleme bei den Mädchen mit Adipositas widersprechen vielen Befunden, sind jedoch vergleichbar mit Ergebnissen von Stachow et al. (2009), die bei Mädchen mit chronisch körperlichen Erkrankungen im mittleren und späten Jugendalter höheres aggressiv-dissoziales Verhalten im SPS-J fanden. Ähnlich zeigten Klasen et al. (2016), dass Mädchen über 13 Jahre im Selbsturteil und über 17 Jahre im Elternurteil mehr ADHS-Symptome als Jungen aufwiesen. Es könnte vermutet werden, dass Mädchen mit Adipositas besonders belastet sind und deswegen mit Problemverhalten versuchen, diese Belastungssituationen $\mathrm{zu}$ verarbeiten. $\mathrm{Zu}-$ künftig muss untersucht werden, ob sich dieser Befund für das mittlere und späte Jugendalter bei klinischen Stichproben replizieren lässt. Zudem ist die ungenügende Zuverlässigkeit der selbstberichteten Verhaltensprobleme $\mathrm{zu}$ berücksichtigen. Übereinstimmend mit früheren Befunden in der Allgemeinbevölkerung konnten noch höhere Ausprägungen in der Hyperaktivität bei den Jungen im Elternurteil festgestellt werden (Deighton et al., 2019; Hölling et al., 2007, 2014).

Ein Altersunterschied konnte nur für die selbstberichteten Verhaltensprobleme statistisch abgesichert werden, die bei den 12- bis 14-Jährigen erhöht waren. Werden frühere Befunde der Allgemeinbevölkerung von Klasen et al. (2016) zu den Störungen des Sozialverhaltens im Elternurteil sowie zu den Verhaltensproblemen des SDQ von Deighton et al. (2019) und Hölling et al. $(2007,2014)$ zusammengefasst, deutet sich ein Peak in der frühen Adoleszenz und ein stetiges Absinken in der späten Adoleszenz an. Erklärungsansätze für die erhöhte Vulnerabilität in der Frühadoleszenz führen aus, dass diese Entwicklungsphase charakterisiert ist durch erhöhte Entwicklungsaufgaben und Alltagsbelastungen bei ungleichem Verlauf in der hormonellen, neuronalen und psychosozialen Entwicklung (Silbereisen \& Weichold, 2018). Um diese Belastungen zu bewältigen, setzen Jugendliche häufiger Problemverhalten ein (vgl. Pinquart \& Silbereisen, 2002). Es kann vermutet werden, dass Jugendliche mit Adipositas im frühen Jugendalter umso mehr belastet 
sind und dementsprechend mehr Problemverhalten einsetzen.

\section{Methodische Einschränkungen}

Obwohl wesentliche bisherige Ergebnisse durch die vorliegende Studie bestätigt werden konnten, unterliegt die Aussagekraft der Befunde einigen methodischen Einschränkungen. Die Studie schloss lediglich Querschnittdaten ein und kann keine Befunde zur Wirkrichtung liefern. Bisherige Ergebnisse legen eine bidirektionale Beziehung nahe (Mannan et al., 2016). Somit sollten zukünftige Studien im Längsschnitt durchgeführt werden, die außerdem noch weitere moderierende Variablen heranziehen. Insgesamt sollte noch der sozioökonomische Status erfasst werden, der sich in vielen Studien als wesentliche Moderatorvariable ergab (Krause et al., 2014). Interessanterweise erwies sich in einer aktuellen Studie der elterliche Berufsstatus als bedeutsamster Prädiktor für psychische Auffälligkeiten (Besser, Döhnert \& Stadelmann, 2019), sodass sich darauf beschränkt werden könnte. Weitere Studien legen einen indirekten Einfluss der Adipositas auf die Entstehung depressiver Symptome nahe (vgl. Sagar \& Gupta, 2018) und weisen der erhöhten Beanspruchung von Jugendlichen mit Adipositas eine vermittelnde Rolle zu (Rankin et al., 2016), sodass die Stressverarbeitungskompetenz noch erhoben werden sollte. Ferner belegten Mediationsanalysen signifikante Effekte für die gewichtsabhängige Stigmatisierung (Blanco et al., 2019) und die Viktimisierung durch Gleichaltrige (van Vuuren et al., 2019). Des Weiteren ist anzumerken, dass unkontrollierte Selektionseffekte aufgrund der klinischen Stichprobe nicht auszuschließen sind und die Fragestellungen zukünftig an einer Stichprobe aus der Allgemeinbevölkerung untersucht werden sollten. Zudem sind die unzulänglichen internen Konsistenzen im SDQ-S anzuführen, die eine Interpretation dieser Befunde einschränken. Schließlich sollten zukünftig zur Erfassung weiterer Facetten internalisierender Prozesse zusätzlich zu dem reliablen und validen Subtest „Ängstlichkeit/Depressivität" des SPS-J-II noch Kennwerte der Emotionsregulation erhoben werden (Koglin, Petermann, Jaščenoka, Petermann \& Kullik, 2013).

\section{Schlussfolgerungen für die klinische Praxis}

Psychische Auffälligkeiten sind bei Adipositas häufig und beeinträchtigen die Compliance. Dies zeigte sich in der Studie von Stachow et al. (2009) vor allem bei Jugendlichen mit Adipositas. Somit wird die Krankheitsbewältigung beeinträchtigt und der Behandlungserfolg ist ge- fährdet. Deswegen muss eine frühzeitige Diagnostik und Intervention erfolgen, um einer Entstehung bzw. Aufrechterhaltung komorbider psychischer Auffälligkeiten vorzubeugen. Hierbei sind insbesondere kognitiv-behaviorale Interventionen zur Steigerung der sozio-emotionalen Kompetenz angezeigt, die vor allem bei Jugendlichen mit Adipositas und geringerer Volitionsstärke gefördert werden sollte (Hampel, Stachow \& Wienert, 2020).

\section{Literatur}

Barkmann, C. \& Schulte-Markwort, M. (2004). Prävalenz psychischer Auffälligkeit bei Kindern und Jugendlichen in Deutschland - ein systematischer Literaturüberblick. Psychiatrische Praxis, 31, 278 287.

Barkmann, C. \& Schulte-Markwort, M. (2012). Prevalence of emotional and behavioural disorders in German children and adolescents: A meta-analysis. Journal of Epidemiology and Community Health, 66, 194-203.

Baumgarten, F., Cohrdes, C., Schienkiewitz, A., Thamm, R., Meyrose, A.-K. \& Ravens-Sieberer, U. (2019). Gesundheitsbezogene Lebensqualität und Zusammenhänge mit chronischen Erkrankungen und psychischen Auffälligkeiten bei Kindern und Jugendlichen: Ergebnisse aus KiGGS Welle 2. Bundesgesundheitsblatt - Gesundheitsforschung - Gesundheitsschutz, 62, 1205-1214.

Becker, A., Wang, B., Kunze, B., Otto, C., Schlack, R., Hölling, H. et al. (2018). Normative data of the self-report version of the German Strengths and Difficulties Questionnaire in an epidemiological setting. Zeitschrift für Kinder- und Jugendpsychiatrie und Psychotherapie, 46, $523-533$.

Besser, A., Döhnert, M. \& Stadelmann, S. (2019). Verschiedene sozioökonomische Faktoren als Prädiktoren für internalisierende und externalisierende Störungsbilder des Kindes- und Jugendalters. Zeitschrift für Kinder- und Jugendpsychiatrie und Psychotherapie, 47, 345-358.

Blanco, M., Solano, S., Alcántara, A. I., Parks, M., Román, F. J. \& Sepúlveda, A. R. (2019). Psychological well-being and weight-related teasing in childhood obesity: A case-control study. Eating and Weight Disorders, 25, $751-759$.

Bühner, M. \& Ziegler, M. (2012). Statistik für Psychologen und Sozialwissenschaftler (3. Aufl.). München: Pearson Studium.

Chao, A. M., Wadden, T. A. \& Berkowitz, R. I. (2019). Obesity in adolescents with psychiatric disorders. Current Psychiatry Reports, 21:3.

Cohen, J. (1988). Statistical power analysis for the behavioural sciences (2nd ed.). Hillsdale: Lawrence Earlbaum Associates.

Deighton, J., Lereya, S. T., Casey, P., Patalay, P., Humphrey, N. \& Wolpert, M. (2019). Prevalence of mental health problems in schools: Poverty and other risk factors among 28000 adolescents in England. The British Journal of Psychiatry, 215, 565-567.

Eschenbeck, H., Kohlmann, C.-W., Dudey, S. \& Schurholz, T. (2009). Physician-diagnosed obesity in German 6- to 14-year-olds. Prevalence and comorbidity of internalising disorders, externalising disorders, and sleep disorders. Obesity Facts, 2, 67-73.

Goldbeck, L., Besier, T., Petermann, F., Karpinski, N. \& Hampel, P. (2007). Validierung des SPS-J an einer kinder- und jugendpsychiatrischen Inanspruchnahmepopulation. Zeitschrift für Psychiatrie, Psychologie und Psychotherapie, 55, 263-270.

Groen, G. \& Petermann, F. (2011). Depressive Kinder und Jugendliche (2. Aufl.). Göttingen: Hogrefe. 
Hampel, P. \& Petermann, F. (2012a). Screening psychischer Störungen im Jugendalter - II (SPS-J-II). Deutsche Adaptation des Reynolds Adolescent Adjustment Screening Inventory (RAASI) (2. Aufl.). Bern: Huber.

Hampel, P. \& Petermann, F. (2012b). Zur Validität des Screenings psychischer Störungen im Jugendalter (SPS-J). Zeitschrift für Psychiatrie, Psychologie und Psychotherapie, 60, 225 - 236.

Hampel, P., Stachow, R. \& Wienert, J. (2020). Psychologische Kennwerte und BMI-SDS bei Jugendlichen mit Adipositas und unterschiedlichen Motivationsstadien in der stationären Rehabilitation. Die Rehabilitation, 59, 157-165.

Hölling, H., Erhart, M., Ravens-Sieberer, U. \& Schlack, R. (2007). Verhaltensauffälligkeiten bei Kindern und Jugendlichen. Erste Ergebnisse aus dem Kinder- und Jugendgesundheitssurvey (KiGGS). Bundesgesundheitsblatt - Gesundheitsforschung - Gesundheitsschutz, 50, 784-793.

Hölling, H., Schlack, R., Petermann, F., Ravens-Sieberer, U. \& Mauz, E. (2014). Psychische Auffälligkeiten und psychosoziale Beeinträchtigungen bei Kindern und Jugendlichen im Alter von 3 bis 17 Jahren in Deutschland - Prävalenz und zeitliche Trends zu 2 Erhebungszeitpunkten (2003 - 2006 und 2009 - 2012). Ergebnisse der KiGGSStudie - Erste Folgebefragung (KiGGS Welle 1). Bundesgesundheitsblatt - Gesundheitsforschung - Gesundheitsschutz, 57, 807 819.

Karow, A., Bock, T., Naber, D., Löwe, B., Schulte-Markwort, M., Schäfer, I. et al. (2013). Die psychische Gesundheit von Kindern, Jugendlichen und jungen Erwachsenen - Teil 2: Krankheitslast, Defizite des deutschen Versorgungssystems, Effektivität und Effizienz von „Early Intervention Services“. Fortschritte der NeurologiePsychiatrie, 81, 628-638.

Klasen, F., Meyrose, A.-K., Otto, C., Reiss, F. \& Ravens-Sieberer, U. (2017). Psychische Auffälligkeiten von Kindern und Jugendlichen in Deutschland. Monatsschrift Kinderheilkunde, 165, 402-407.

Klasen, F., Petermann, F., Meyrose, A.-K., Barkmann, C., Otto, C., Haller, A.-C. et al. (2016). Verlauf psychischer Auffälligkeiten von Kindern und Jugendlichen. Kindheit und Entwicklung, 25, 10-20.

Koglin, U., Petermann, F., Jaščenoka, J., Petermann, U. \& Kullik, A. (2013). Emotionsregulation und aggressives Verhalten im Jugendalter. Kindheit und Entwicklung, 22, 155-164.

Krause, L., Kleiber, D. \& Lampert, T. (2014). Psychische Gesundheit von übergewichtigen und adipösen Jugendlichen unter Berücksichtigung von Sozialstatus und Schulbildung. Prävention und Gesundheitsförderung, 9, 264-273.

Lambert, M., Bock, T., Naber, D., Löwe, B., Schulte-Markwort, M., Schäfer, I. et al. (2013). Die psychische Gesundheit von Kindern, Jugendlichen und jungen Erwachsenen - Teil 1: Häufigkeit, Störungspersistenz, Belastungsfaktoren, Service-Inanspruchnahme und Behandlungsverzögerung mit Konsequenzen. Fortschritte der Neurologie-Psychiatrie, 81, 614-627.

Lohbeck, A., Schultheiß, J., Petermann, F. \& Petermann, U. (2015). Die deutsche Selbstbeurteilungsversion des Strengths and Difficulties Questionnaire (SDQ-Deu-S). Diagnostica, 61, 222-235.

Mannan, M., Mamun, A., Doi, S. \& Clavarino, A. (2016). Prospective associations between depression and obesity for adolescent males and females - A systematic review and meta-analysis of longitudinal studies. PloS ONE, 11, e0157240.

Pinquart, M. (2017). Psychische Gesundheit von chronisch körperlich kranken Kindern und ihren Eltern - Ergebnisse von Metaanalysen. Praxis der Kinderpsychologie und Kinderpsychiatrie, 66, 656-671.

Pinquart, M. \& Shen, Y. (2011a). Anxiety in children and adolescents with chronic physical illnesses: A meta-analysis. Acta Paediatrica, 100, $1069-1076$.

Pinquart, M. \& Shen, Y. (2011b). Depressive symptoms in children and adolescents with chronic physical illness. An updated meta-analysis. Journal of Pediatric Psychology, 36, 375-384.
Pinquart, M. \& Silbereisen, R. K. (2002). Gesundheitsverhalten im Kindes- und Jugendalter. Bundesgesundheitsblatt - Gesundheitsforschung - Gesundheitsschutz, 45, 873-878.

Quek, Y.-H., Tam, W. W. S., Zhang, M. W. B. \& Ho, R. C. M. (2017). Exploring the association between childhood and adolescent obesity and depression: A meta-analysis. Obesity Reviews, 18, $742-$ 754.

Rankin, J., Matthews, L., Cobley, S., Han, A., Sanders, R., Wiltshire, H. D. et al. (2016). Psychological consequences of childhood obesity: Psychiatric comorbidity and prevention. Adolescent Health, Medicine and Therapeutics, 7, 125-146.

Ravens-Sieberer, U., Klasen, F. \& Petermann, F. (2016). Psychische Kindergesundheit. Kindheit und Entwicklung, 25, 4-9.

Rossmann, P. \& Pichler-Janisch, P. (1998). Zur Mutter-Kind-Übereinstimmung bei der Beurteilung von Depressivität, Neurotizismus und Extraversion von Kindern mittels DTK und HANES-KJ. Diagnostica, 44, $182-188$.

Sagar, R. \& Gupta, T. (2018). Psychological aspects of obesity in children and adolescents. Indian Journal of Pediatrics, 85, 554-559.

Stachow, R., Kiera, S., Tiedjen, U. \& Petermann, F. (2009). Chronisch kranke Jugendliche. Compliance und psychische Symptome. Monatsschrift Kinderheilkunde, 157, 1141-1146.

Silbereisen, R. K. \& Weichold, K. (2018). Jugend (12-19 Jahre). In W. Schneider \& U. Lindenberger (Hrsg.), Entwicklungspsychologie (8., vollst. überarb. Aufl., S. 239-263). Weinheim: Beltz.

Thapar, A., Collishaw, S., Pine, D. S. \& Thapar, A. K. (2012). Depression in adolescence. The Lancet, 379, 1056-1067.

van Vuuren, C. L., Wachter, G. G., Veenstra, R., Rijnhart, J. J. M., van der Wal, M. F., Chinapaw, M. J. M. et al. (2019). Associations between overweight and mental health problems among adolescents, and the mediating role of victimization. BMC Public Health, 19, 612.

Wang, S., Sun, Q., Zhai, L., Bai, Y., Wei, W. \& Jia, L. (2019). The prevalence of depression and anxiety symptoms among overweight/obese and non-overweight/non-obese children/adolescents in China: A systematic review and meta-analysis. International Journal of Environmental Research and Public Health, 16(3): 340. https://doi. org/10.3390/ijerph16030340

Woerner, W., Becker, A., Friedrich, C., Klasen, H., Goodman, R. \& Rothenberger, A. (2002). Normierung und Evaluation der deutschen Elternversion des Strengths and Difficulties Questionnaire (SDQ). Ergebnisse einer repräsentativen Felderhebung. Zeitschrift für Kinder- und Jugendpsychiatrie und Psychotherapie, 30, 105-112.

\section{Prof. Dr. Petra Hampel}

Institut für Gesundheits-, Ernährungs- und Sportwissenschaften Europa-Universität Flensburg

Auf dem Campus 1

24943 Flensburg

petra.hampel@uni-flensburg.de

\section{Prof. Dr. Franz Petermann}

Zentrum für Klinische Psychologie und Rehabilitation der Universität Bremen

Grazer Straße 6

28359 Bremen

\section{Dr. Rainer Stachow}

Ärztlicher Direktor

Ostseeklinik Grömitz

Blankwasserweg 65

23743 Grömitz 